\title{
LXXVII. A new radiant emission from the spark
}

\section{R.W. Wood}

To cite this article: R.W. Wood (1910) LXXVII. A new radiant emission from the spark, Philosophical Magazine Series 6, 20:118, 707-712, DOI: 10.1080/14786441008636956

To link to this article: http://dx.doi.org/10.1080/14786441008636956

册 Published online: 21 Apr 2009.

Submit your article to this journal 정

Џlll Article views: 3

Q View related articles ¿

4 Citing articles: 2 View citing articles $\square$ 
of $\lambda$ is accordingly

$$
\frac{\mathrm{D}}{\mathrm{T}}=\sqrt{\frac{\bar{\lambda}}{\mathrm{T}}}
$$

it thus varies inversely as the square root of the length of the interval of time. 'This result is of the same form as the classical one used by E. v. Schweidler in the paper referred to earlier.

The probable value of $|\mathrm{N}-\lambda \mathrm{T}|$ (der durchschnittlicher Fehler) is much more difficult to calculate.

LXXVII. A New Liadiant Emission from the Spark. By R. W. Woon, Professor of Experimental Physics in the Joluns Hopkins University*.

\section{[Plate XIV.]}

I SCARCELY know how to designate the peculiar type of radiation referred to in the present paper, which was first discovered over two years ago in the course of some experiments made with a view of ascertaining whether the Schumann waves from the spark gave rise to any fluorescence of the air by which they were absorbed. It is now known that there is a feeble ultra-violet luminosity of air or nitrogen gas surrounding a small mass of radium, in other words the radium renders the gas luminescent. To test for a fluorescence dne to the absorption of very short lightwaves, the condenser spark between aluminium electrodes was passed behind and very close to a vertical strip of metal which completely concealed the spark, but which enabled observation, either visual or photographic, of the air in its immediate vicinity. If the air in the room was free from dust and smoke absolutely nothing could be seen with the eye, even after prolonged resting in the dark. A photograph, however, made with a smail camera provided with a quartz lens, showed that the air around the spark was a source of a powerful actinic radiation, which was completely stopped by the intervention of a glass plate between the camera and the spark. The first photograph of the phenomenon which was obtained is reproduced on Pl. XIV. fig. 6. The narrow strip of metal between the two wider strips was about $1 \mathrm{~cm}$. in width; the spark discharge was concealed behind this.

Two hypotheses immediately presented themselves: (a) we are dealing with a scattering of the shortest waves by the

* Communicated by the Author. 3 A $\dot{2}$ 
air molecules or microscopical dust particles, as in case of blue-sky; $(b)$ ultra-violet fluorescence of the air caused by the absorption of the Schumann waves. As a matter of fact neither hypothesis turned out to be tenable, but I mention them to show that they have been carefully considered.

If the emission of ultra-violet light by the air was merely a scattering, its spectrum should be identical with that of the spark : if, on the contrary, it is a fluorescence phenomenon, its spectrum would be totally different. In the spectroscopic work it was necessary to get as cluse as possible to the spark, and yet run no risk of having its direct light enter the slit of the instrument. To meet this requirement the apparatus shown in fig. 1 was constructed. A disk of,

Fig. 1.

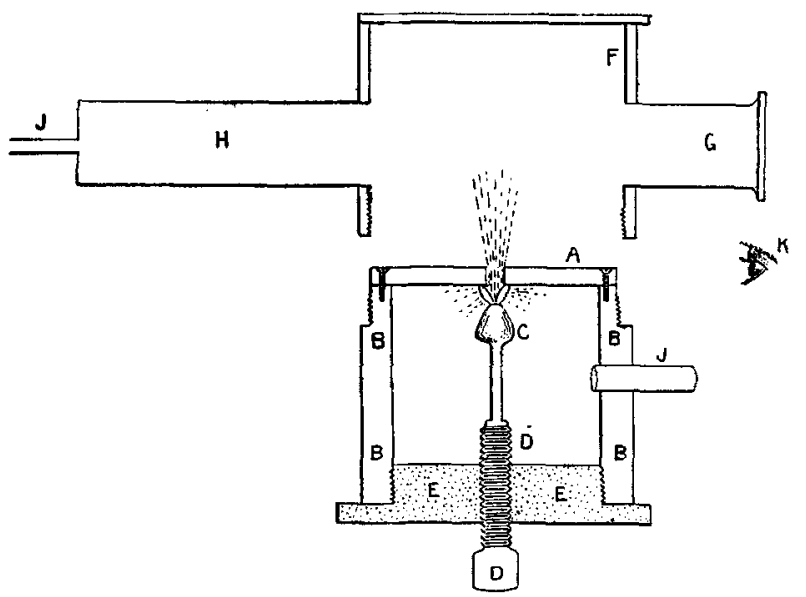

aluminium, $3 \mathrm{~mm}$. thick, was perforated with a hole $1.5 \mathrm{~mm}$. in diameter and fastened to a short brass cylinder B. The aluminium electrode $\mathrm{C}$ was carried by a screw $\mathrm{D}$, which passed through the ebonite cap $\mathrm{E}$. The spark discharge passed between $\mathrm{C}$ and the inner rim of the bole in the aluminium disk. If the spark chamber is hermetically sealed the explosive expansions of the air are apt to force the spark aureole, which is pale green in the case of the aluminium spark, out through the hole. The small lateral tube $J$ prevents this, and serves as well for the introduction of various gases. The length of the spark must be so adjusted that no visible portion is forced out through the hole, when viewed from the position $\mathrm{K}$.

A second brass cylinder F, closed at the top and fitted 
with two lateral tubes $H$ and $G$, can be screwed to the spark chamber when it is desired to study the emission in dry filtered air or some other gas. The tube $G$ is closed with a quartz window, while $H$ terminates in a smaller tube $J$ for the introduction of gas. The emission is quite invisible in dustfree air, yet it can be photographed with an exposure of one or two minutes with a quartz lens of $2 \mathrm{~cm}$. aperture and $15 \mathrm{~cm}$. focus. In arranging the position of the quartz camera the focal plane should be examined with an eyepiece in a dark room to make sure that no part of the lens receives light from the edge of the bole; in other words, the top of the lens must be just below the plane of the aluminium disk $A$.

In order to get an idea of the appearance of scattered light, the air around the apparatus was filled with smoke and the spark discharge started. The photograph obtained in this way is reproduced on Pl. XIV. fig. 1. Fig. 2 was obtained when the air was free from smoke or dust, and shows the appearance of the emission with which we are concerned. A comparison of these two photographs shows us at once that the emission does not extend nearly so far out from the aperture as does the luminous region of lightscattering smoke. It appears as if it were rapidly absorbed by the air. That this is not due to differences in the time of exposure is shown by the original negatives, for fig. 2 has a density nearly double that of fig. 1 in the immediate proximity of the aperture in the disk. An experiment was next made to ascertain the nature of the light given out by the emission. A fine thread of fused quartz, about $2 \mathrm{~mm}$. in length, was mounted at the edge of the aperture by means of a small arop of soluble glass. This scattered the light of the spark, forming a narrow linear source of spark light located at the centre of the base of the emission. The slit tube of a small quartz spectrograph was removed, and the luminous quartz thread brought into its place. The resulting photograph is shown in fig. 3, a continuous band of light, the spectrum of the quartz fibre, with the emission above it and about at its centre. 'This picture proved that the light given out by the emission embraced a limited range of wave-lengths in the region 300-310. This picture was secured with an exposure of only fifteen minutes, which made it seem probable that the spectrum of the emission could be obtained with a fairly narrow slit. Fig. 5 shows a spectrogram obtained with a wide slit, the aluminium lines showing faintly as a result of diffused light : the lower spectrum is that of the spark for comparison. The spectrum of the emission consists of two broad bands, one very strong, the other (to the right) much 
weaker. These were found to be identical with the so-called "water bands" of the oxy-hydrogen flame, as is clearly brought out by fig. 9 , in which the upper spectrum is that of the oxy-hydrogen flame, the lower that of the emission. In addition to these bands I obtained on one plate lines it wave-lengths 3576,3537 , and 3369 , which are identical with lines attributed to nitrogen in the spectrograms published by Eder and Valenta of the spark between wet carbon electrodes. There is, in addition, a line which is imbedded in the water-band, as shown in figs. 7 and 8 . In fig. 7 the upper spectrum is that of the oxy-hydrogen flame (overexposed), below it the aluminium spark, and at the bottom the spark emission. The nitrogen lines come out very clearly in this case. The spectrum by Eder and Valenta, which is practically identical with that of the emission, was obtained by passing the discharge of an induction-coil between wet carbon electrodes, and differs from that of the oxy-hydrogen flame in that it shows the nitrogen lines above referred to.

It looked very much as if the emission might be due to the fluorescence of nitrogen and water vapour, resulting from the absorption of the Schumann waves; this would explain its failure to penetrate the air to any considerable distance. To test this point the auxiliary tube was attached to the spark chamber, the emission being studied through the quartz window attached to the tube G. The apparatus was first filled ' with air carefully dried by passage througlı a tube filled with phosphorus pentoxide, and then with air passed through a plug of wet cotton. The emission was photographed in each case, but no difference in the intensity of the images could be detected. Oxygen and nitrogen were then tried in succession. In the former there was almost no trace of the emission, while in the latter it was much brighter and extended to a greater distance from the aperture than in air. Photographs of the phenomenon in these two gases are reproduced on PI. XIV. fig. 4. The emission is photographed against the very black background furnished by the long tube $\mathrm{H}$, in fig. 1. The time of exposure was the same in each case, and the two plates were developed together. The aperture is to the right in each picture, the emission shooting out towards the left. The crescent of light is the inner $\theta$ dge of the tube $\mathrm{H}$ illuminated by diffused light.

The next question was to determine whether the presence of oxygen prevented the formation of the emission, or whether the gas exerted an absorbing action. This was a difficult matter to deternine, since numerous experiments showed that no substance was transparent to the emission. 
A plate of white fluorite, $0.5 \mathrm{~mm}$, in thickness, which had heen found very transparent for the Schumann waves by Dr. Lyman, who very kindly placed it at my disposal, together with an end-on bydrogen tube for the production of Schumann waves, when placed over the aperture was found to destroy all trace of the emission. This disposed of the theory that we were dealing with a fluorescence produced by the short waves. Thin aluminium foil, such as is used with the Lenard tubes, was found to be equally opaque. It is therefore a difficult matter to start the emission in a given gas and pass it into a different one. The problem was finally solved by an experiment designed to test one of the theories that I had evolved to explain the phenomenon. It occurred to me that we might be dealing with hydrogen ions, shot off from the electrodes, which, by combination with the oxygen of the air, gave rise to a spectrum similar to that of the oxyhydrogen flame. We might in this way explain the lessened effect in oxygen as a result of the circumstance that the "combustion" of the ions took place almost entirely within the small tube with which the disk of aluminium was perforated. If this were the case, it seemed probable that if the emission were formed in air, and a small jet of oxygen were directed across it transversely, we should observe a more intense action at the point where the emission met the oxygen jet. The experiment was tried, and it was found that the gas jet merely interrupted the emission, killed it in other words, precisely as if it absorbed it. If the emission was started in air and a jet of nitrogen blown gently against the aperture, the emission was found to shoot out much farther and to be of greater intensity. The magnetic field appeared to be without action on it, though the experiment was found to be attended with difficulties on account of the action of the magnet on the spark.

It is still more difficult io study the action of an elfcctrostatic field. The material constituting the emission is evidently shot from the aperture at a very high velocity, for it is impossible to blow it aside with a strong jet of air ; moreover, if air is forced continuously into the auxiliary chamber, passing through the aparture in the aluminium disk at a high velocity, the emission does not appear to be held back in the slightest degree.

I am unable to explain its reactions with oxygen and nitrogen, and the apparent failure of the presence or absence of water vapour to modify the intensity of the spectrum, which is made up chietly of the so-called water-bands. These bands appear when hydrogen burns in oxygen, and yet 
oxygen destroys the luminosity of the emission. This fact appears to be of the greatest importance in connexion with the origin of these bands. If I remember rightly, the introduction of chlorine gas into a sodium flame destroys its emission of the $\mathrm{D}$ lines, and there may be some analogy between the two phenomena. I intend sometime to photograph the spark directly utilizing the principle of the spectroheliograph. An image of the spark obtained with monochromatic light of the wave-length of the water-band may tell us something about the origin of the emission. In the meantime I hope that some study of the phenomenon will be made by others, as it appears to be of considerable importance in connexion with the origin of radiation.

It seems quite likely that the "Entladungsstrahlen" may be identical with the emission, for they are absorbed by oxygen. One great difficulty in the investigation is the apparent impossibility of separating the emission from the ultra-violet and visible light which goes out with it.

\section{Some Experiments on Refraction by non-homogeneous Media. By R. W. Wood*.}

[Plate XIV. fig. 10.]

T THAT the apparent diameter of a body surrounded by a 1 refracting atmosphere is slightly larger than its true diameter is well known. An extreme case is the mercury thread of a thermometer. At the other extreme we have the earth as seen from the moon.

This magnification by a non-homogeneous atmosphere, in which there is no sharply defined refracting surface (as in the case of the earth's atmosphere) can be very nicely shown in the following way :-

Make a small rectangular glass tank by cementing five squares of glass together with sealing-wax. Fill it with melted gelatine and support an empty test-tube in the fluid with a clamp stand. The bottom of the test-tube should be within half a centimetre of the bottom. After the jelly bas solidified, pour hot water into the test-tube, and immediately withdraw it. It will leave a cylindrical hole in the jelly, with a hemispherical bottom. Now pour a mixture of glycerine and powdered chalk into the cavity until it is half full. Fill the remainder with water to which a few drops of milk have been added. The glycerine will gradually

* Communicated by the Author. 
WooD.

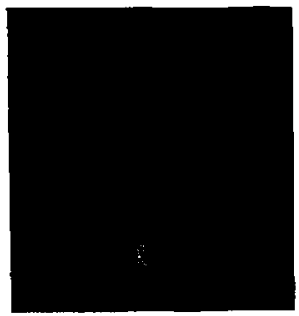

FIr. 1.
Phil. Mag. Ser. 6, Vol. 20, Pl. XIV.

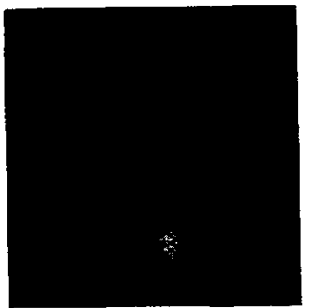

Fig. 2.

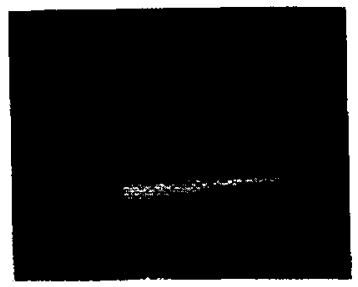

Fig. 3.
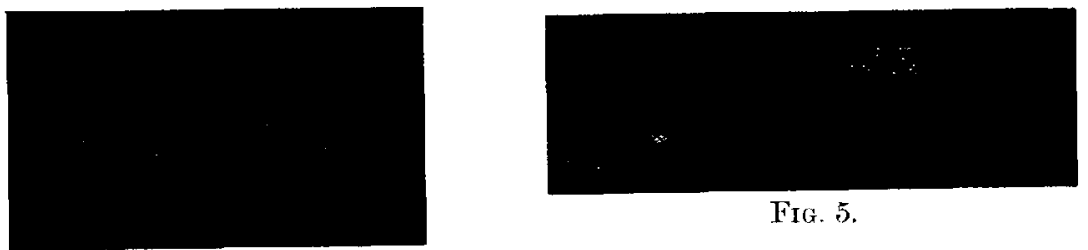

FIG. 5.

FIG. 4.

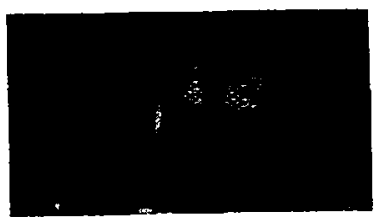

FIG. 6.

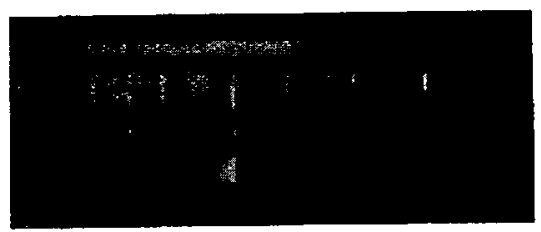

FI(x. 7 .
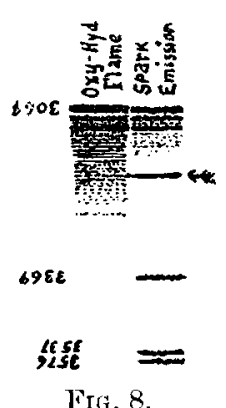

a

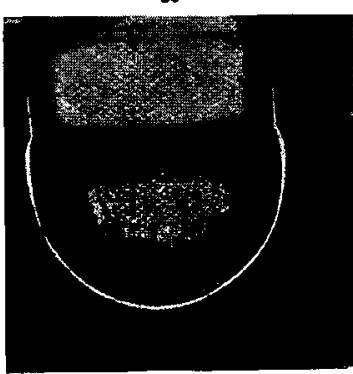

FIG. 10.

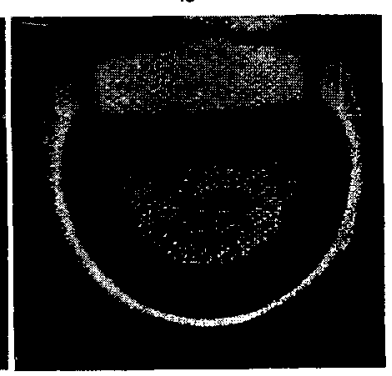

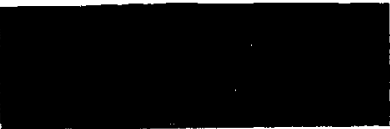

FIt. 9. 